\title{
Robust boundary detection and tracking of left ventricles on ultrasound images using active shape model and ant colony optimization
}

\author{
Yaonan Zhang ${ }^{\mathrm{a}, *}$, Yuan Gao ${ }^{\mathrm{a}}$, Jinling Jiao ${ }^{\mathrm{a}}$, Xian $\mathrm{Li}^{\mathrm{a}}$, Sai $\mathrm{Li}^{\mathrm{a}}$ and Jun Yang ${ }^{\mathrm{b}}$ \\ ${ }^{a}$ Sino-Dutch Biomedical and Information Engineering School, Northeastern University, Shenyang, \\ China \\ ${ }^{b}$ Department of Cardiovascular Ultrasound, The First Hospital of Chinese Medical University, \\ Shenyang, China
}

\begin{abstract}
Information regarding the motion, strain and synchronization are important for cardiac diagnosis and therapy. Extraction of such information from ultrasound images remains an open problem till today. In this paper, a novel method is proposed to extract the boundaries of left ventricles and track these boundaries in ultrasound image sequences. The initial detection of boundaries was performed by an active shape model scheme. Subsequent refinement of the boundaries was done by using local variance information of the images. The main objective of this paper is the formulation of a new boundary tracking algorithm using ant colony optimization technique. The experiments conducted on the simulated image sequences and the real cardiac ultrasound image sequences shows a positive and promising result.
\end{abstract}

Keywords: Active shape model, image segmentation, boundary tracking, ant colony optimization, motion estimation

\section{Introduction}

Echocardiography is one of the major imaging techniques to measure the heart functions [1]. The detection and tracking of the cardiac boundaries, especially left ventricular (LV) boundaries, on echocardiography images are crucial for the quantitative cardiac functional assessment. This task remains a challenging problem due to several reasons: 1) Ultrasound is one of the most noisy methods among common medical imaging techniques, and secondly it is difficult to distinguish between ultrasound speckles and noisy images during image processing and analysis; 2) the cardiac motions are large due to fast motion of the heart muscle and respiratory interferences; and 3) the cardiac boundaries are not necessarily close because of the dynamic opening and closing of mitral valves.

\footnotetext{
${ }^{*}$ Corresponding author: Yaonan Zhang, Sino-Dutch Biomedical and Information Engineering School, Northeastern University, No. 11, Lane 3, Wenhua Road, Shenyang 110819, China. Tel.: +86-24-83685261; Fax: +86-24-83681955; E-mail: zhangyn@bmie.neu.edu.cn.
} 
Very little work has been done and reported in this field of research [2-6]. At the beginning, "deformable model" based segmentation techniques was used like level set and snake techniques $[2,3]$. This kind of method has some drawbacks: 1) the final results depend heavily on the initial contours that are often drawn manually, which makes the whole procedure difficult being full-automatic and repeatable; 2) the cardiac boundaries are often vague and noisy, which make it difficult to formulate the boundary edges into an analytic energy function. Another generic method is the optical flow method [4], which is good at generating pixel-wise motion vectors with sub-pixel accuracy, but it is sensitive to noises. Consequently the low repeatability of the algorithm makes it difficult to be used clinically. More recent work used the unified framework for fusing motion estimates from multiple appearance models and fusing a subspace shape model with the system dynamics and measurements with heteroscedastic noise $[5,6]$.

In this paper, the initial detection of boundaries was performed by an active shape model (ASM) scheme. ASM's advantage is that it is not based on any kind of theoretical analytic model, rather it is based on a statistical model, automatically learn from training data set. It also builds the local image features into appearance model, which is quite robust against noises. This method is used here to detect the initial cardiac boundaries.

For the boundary tracking problem, many similar methods have been used in the literature, e.g. shape tracking, contour tracing etc. The shape tracking in the articles $[5,6]$ was related to the tracking of "control points" of cardiac boundaries in an image sequence. In this paper, the word "boundary tracking" is used to track the movement of each pixel along a cardiac boundary in an image sequence. This sort of tracking could result into a measurement of a local deformation along a cardiac boundary in continuous heart cycles, which can be used to assess the functions of a particular cardiac chamber. For this a novel tracking method is proposed based on ant colony optimization. Because of the space limitation, the detection and tracking results only for the left ventricles are shown in this paper.

\section{The proposed method}

\subsection{Initial cardiac boundary detection by active shape model}

Active Shape Model (ASM) method [7-9] has been used successively in the segmentation of medical images for many organs, such as brain and cardiac structures. ASM is a statistical model for the shape of objects that iteratively change to fit onto the corresponding objects in a new image. Constructing a statistical shape model consists of extracting the mean shape and a number of modes of variation from a collection of training samples. In this work, the training data are manually generated from 20 image sequences. A principal component analysis was used to build active shape models and active appearance models. During the segmentation phase, a Mahalanobis distance is used in the feature selection. This procedure generates the initial boundaries for cardiac chambers.

\subsection{Boundary refinement based on image variance}

ASM segmentation method is relatively a successful method, but it has some limitations. For example, ASM heavily depends on the training data which are manually generated. The position inaccuracy of the landmarks and inconsistency of the landmarks between different images can have a big impact on the ASM segmentation results. Moreover, the local shape and image details on the target image are often different with the training images. Therefore, a refinement procedure is proposed as follows. 


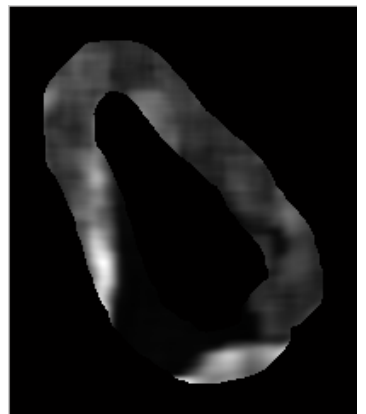

(a)

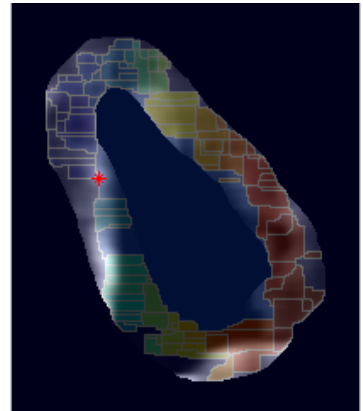

(b)

Fig. 1. (a) The original variance distribution of neighboring contour area for the left ventricle based on real Ultrasound images; (b) The ridge line extracted directly by a watershed algorithm based on the variance distribution.

Image variance reflects the degree of local texture information deviating from the regional average gray level. The introduction of the variance information could provide a strong driving force for cardiac chamber region segmentation. Refining the initial ASM contours is a process of finding the accurate edge of the region in a neighboring contour area based on image local variance information.

By calculating the variance distribution of neighboring contour areas, the initial distribution image of variance is obtained, as illustrated in Figure 1(a). Detecting edges in a geographic data is to find the highest closed ridge line (also called watershed) in a region, therefore a watershed algorithm can generate a good image watershed. However, due to the rich details of the ultrasound cardiac images and the influence of the speckle \& noises, the watershed obtained through a watershed algorithm normally results in a number of local small waterline rings adjacent to each other, as shown in Figure 1(b).

In order to obtain a closed ridge line of the left ventricle, the ridge image is turned into an undirected graph, with each pixel as a node and the edges between two nodes weighted by the average gray level of the two pixels (i.e. the average of the variance values). In order to find the highest ridge line, the reciprocal of the initial weights is used (Figure 2(a)), and the peak ridge line can be obtained by a shortest path algorithm, and a result is illustrated in Figure 2(b).

The refinement is an iteration process, which is illustrated in Figure 3 with the initial boundary line obtained from an ASM algorithm.

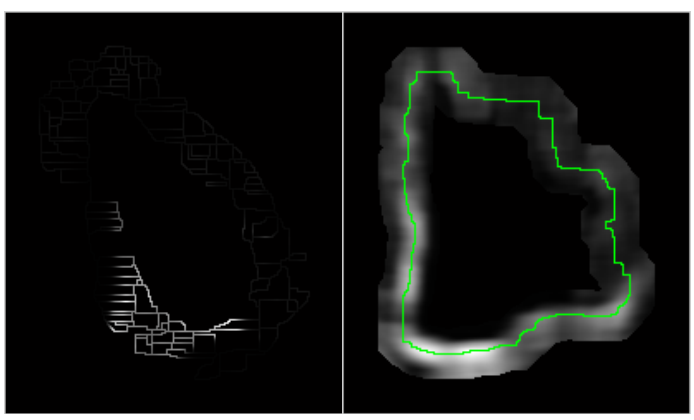

(a)

(b)

Fig. 2. (a) The reciprocal of the initial weights; (b) The final peak line. 


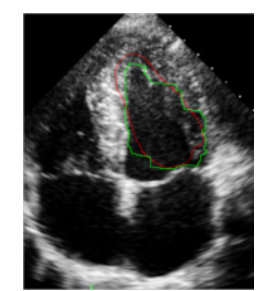

(a)

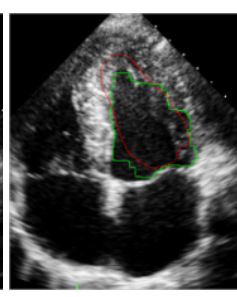

(b)

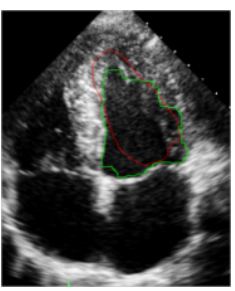

(c)

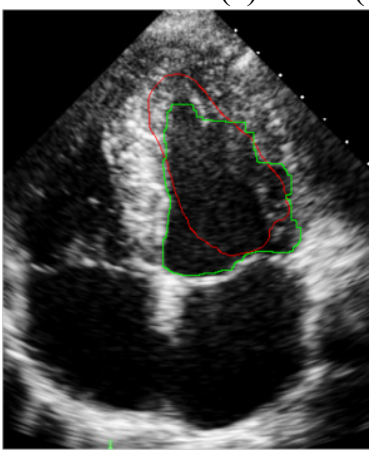

(d)

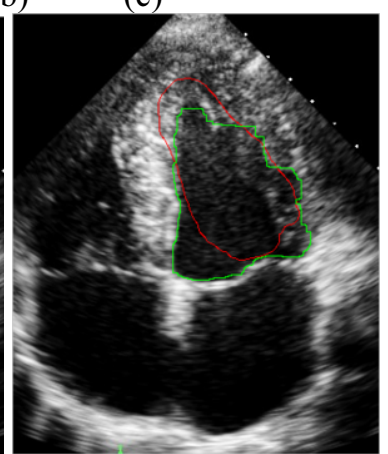

(e)

Fig. 3. The 5 iteration refinement process (green) comparing with original ASM contour line (red); (a)-(e) is corresponding to the contour line of refinement results from iteration 1 to 5 .

\subsection{The cardiac boundary tracking by ant colony optimization}

In order to track a cardiac boundary in an ultrasound image sequence, multiple knowledge sources is used, such as motion vector constraints along sequence (temporal constraints), local similarity of motion vectors (spatial constraints) etc. Ant Colony Optimization (ACO) [10] is a new method which can be incorporated in this problem. In our previous work [11,12], ACO has been used to improve the block matching for motion estimation. A new formulation is used for the cardiac boundary tracking problem, which is elaborated in the following.

For each point $P_{i}(k)$ on the boundary contour of the current frame (say frame $i$ ), there has been a number of candidate pixels on the corresponding contour of the next frame (frame $i+1$ ) within a neighborhood scope. There is a candidate motion vector $u=P_{(i+l), k}(j)-P_{i}(k)$ with every candidate point $P_{(i+1), k}(j)\left(j=1,2, \ldots, N_{C}, N_{C}\right.$ is the number of candidate points). In the framework of ACO, the process of motion estimation is formulated as the process of selecting the candidate that makes the transition probability achieving maximum as follows:

$$
M=\arg \max _{u}\{p(u)\} .
$$

The transition probability related to a candidate motion vector can be calculated by the following equation:

$$
p(u)=\frac{[\tau(u)]^{\alpha}[\eta(u)]^{\beta}}{\sum_{v \in \Gamma}[\tau(v)]^{\alpha}[\eta(v)]^{\beta}}
$$


where $\Gamma$ is the candidate motion vector space, $\tau(u)$ is the pheromone, $\eta(u)$ is the heuristics, $\alpha$ is the pheromone restriction factor, and $\beta$ is the heuristic restriction factor.

$\eta(u)$ is calculated as

$$
\eta(u)=\frac{\lambda_{1}}{\varepsilon(u)+\varepsilon_{0}}+\lambda_{2} F\left(P_{i}(k), P_{(i+1), k}(j)\right)
$$

where $\varepsilon(u)$ is the similarity measure value between two candidate matching points, and $\varepsilon(u)$ can be calculated according to a similarity measure functions like SAD (Sum of Absolute Difference) or cross-correlation coefficient. In this paper, SAD is chosen as the similarity measure function. $\varepsilon_{0}$ is a balance factor (a constant value) used to avoid zero division (e.g. for a zero similarity measure).

$\lambda_{1}$ and $\lambda_{2}$ are the weighting factors and need a dynamic adjustment in order to deal with complex heart movement. When the current search location is full of details, the ratio $\lambda_{1} / \lambda_{2}$ needs to be larger, this means that the heuristic now mainly depends on similarity measurement. In another case when the current searching region contains fewer details, the ratio $\lambda_{1} / \lambda_{2}$ is set to be lower value, which indicates the heuristic mainly depends on the constraint of the contour line.

In Eq. (3), $F($.$) is a contour dependency constraint. In this paper, the contour dependency con-$ straint is selected as a Gaussian function:

$$
F(d)=e^{-\frac{d^{2}}{2 \sigma^{2}}}
$$

where, $\sigma$ is the standard deviation of the Gaussian function, and $d$ is defined as the distance from the motion searching point at current frame to the closest point at the corresponding contour line of the next frame.

The updating of the pheromone is done at the end of each iteration process, and the pheromone related to every candidate motion vector will be updated as

$$
\tau(u) \leftarrow \rho \cdot \tau(u)+\gamma_{1} \cdot \Delta \tau_{1}(u)+\gamma_{2} \cdot \Delta \tau_{2}(u)
$$

where $\Delta \tau_{1}(u)$ is the pheromone increment of space constraints, which is calculated according to the pheromone of best motion vectors related to surrounding searching points. $\Delta \tau_{2}(u)$ is the negative incremental pheromone designed to avoid multi-matching problem. It is a punishment mechanism that will guide the motion vector to a unique and accurate candidate point. In this paper, the amount of the negative incremental of pheromone is linked to the numbers of multi-matching vectors.

\section{The results and discussion}

The proposed methods have been tested on many ultrasound image sequences, some of them are simulated images where the accuracy of estimated motion vectors can be easily validated, and some of 
the experiments are based on real ultrasound images taken from Ultrasound diagnosis machines in hospitals. Figure 4 illustrates the results on a simulated Ultrasound image sequence. Figure 4(a) shows the estimated motion vectors from the algorithm as proposed above and Figure 4(b) depicts the comparison of the estimated motion amplitude and the "true" amplitude for the simulated ultrasound images. The two close curves indicate that the proposed algorithm works well for the simulated images.

Figure 5 shows the results on a real clinical Ultrasound image sequence. This sequence was taken from Philips iE33 Ultrasound machine on a real patient. Figure 5(a) illustrates the estimated motion vectors and Figure 5(b) plots the motion amplitude curve along several heart beat cycles. It is obvious that the direction of the vectors is pointing to the center of the left ventricular region because the image pair relates to the systolic period. It is smooth on the whole, and there is no abrupt vector. From the local zooming figure (left bottom figure of Figure 5(a)) it is easily seen that the motion vectors are pointing in the right direction clearly and consistently, and no crossing and overlapping occurs. Figure 5(b) demonstrates that the motion amplitude fits the motion of the heart very well. Further research is carried out on the clinical usages of this kind of curves.

\section{Conclusion}

The detection and tracking of the cardiac boundaries, especially left ventricular (LV) boundaries, on echocardiography images remains a challenging task. Some of the novel methods have been proposed in this paper. An active shape model algorithm is chosen to detect the initial contours for cardiac boundaries. In order to get more accurate boundary contours, a refinement algorithm is proposed based on the local image variances. For the boundary tracking problem, a new method is proposed using ant colony optimization techniques. The experiments carried out on the simulated image sequences and the real cardiac ultrasound image sequences and the output shown a positive and promising result. It should be pointed out that the proposed method still needs refinements, such as: 1) how to handle the inaccuracy of landmarks during ASM training; 2) the classification of different ASM models for different classes of patients; 3 ) the contour refinement process might be better in order to combine with other image features; 4) more elaborated comparison of the proposed boundary tracking algorithm with other methods.

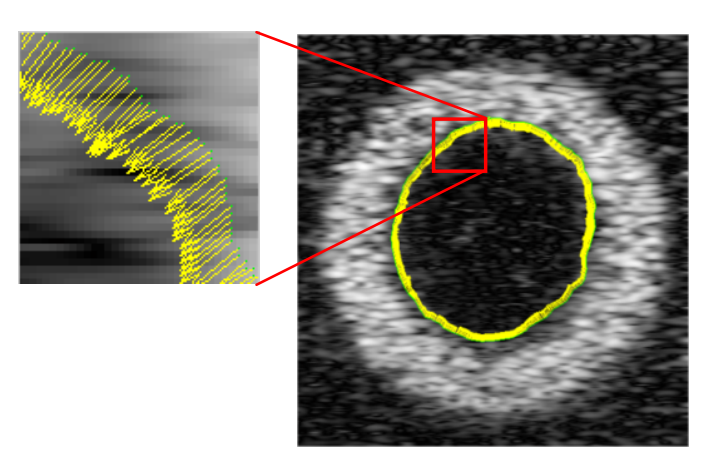

(a)

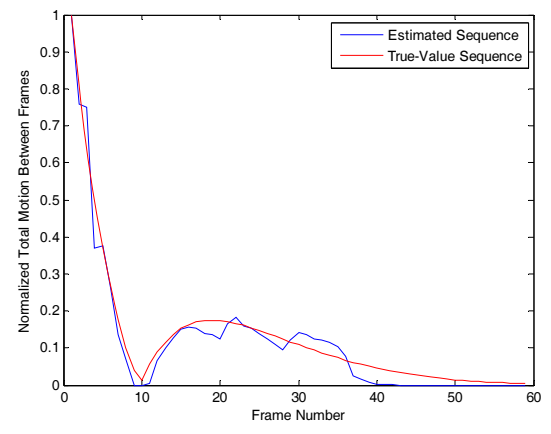

(b)

Fig. 4. (a) The estimated motion vectors on the simulated Ultrasound images; (b) The comparison of the estimated motion amplitude and the "true" amplitude for the simulated Ultrasound images. 


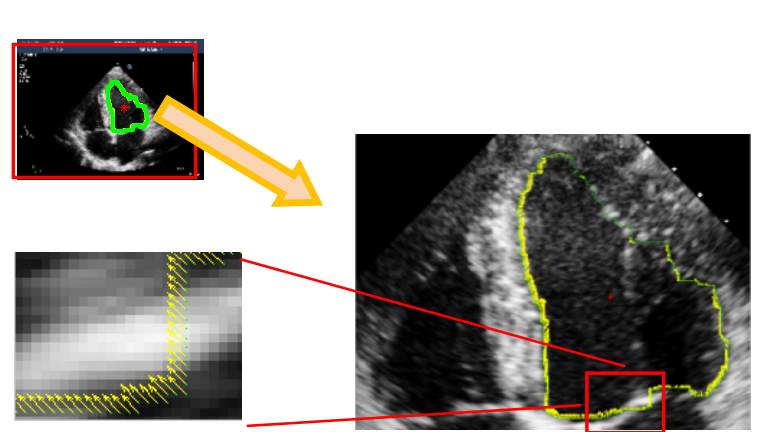

(a)

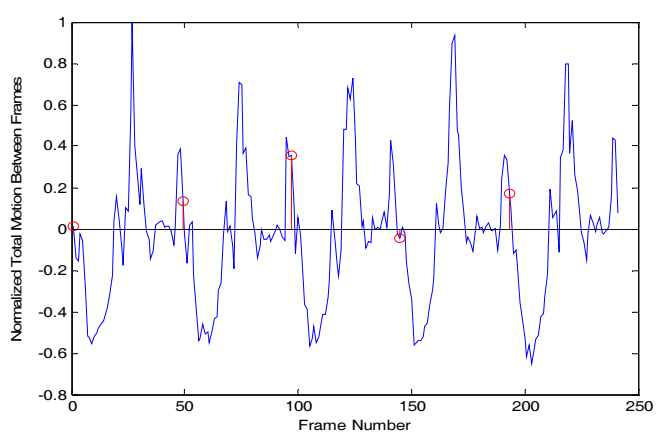

(b)

Fig. 5. (a) The estimated motion vectors of the left ventricle of real clinical Ultrasound images; (b) The left ventricle motion amplitude curve along several heart beat cycles.

\section{Acknowledgement}

This work is sponsored by Natural Science Foundation (61372014) of China and Natural Science Foundation of Liaoning Province (Project 201202071), China.

\section{References}

[1] R. Jasaityte, B. Heyde and J. D'hooge, Current state of three-dimensional myocardial strain estimation using echocardiography, J. Am. Soc. Echocardiogr. 26 (2013), 15-28.

[2] V. Chalana, D.T. Linker, D.R. Haynor and Y. Kim, A multiple active contour model for cardiac boundary detection on echocardiographic sequences, IEEE Transactions on Medical Imaging 15 (1996), 290-298.

[3] G. Jacob, J.A. Noble, C. Behrenbruch, A.D. Kelion and A.P. Banning, A shape-space-based approach to tracking myocardial borders and quantifying regional left-ventricular function applied in echocardiography, IEEE Transactions on Medical Imagin 21 (2002), 226-238.

[4] P. Wang, S.H. Zhou and M. Szucs, Endocardium tracking by fusing optical flows in straightened images with learning based detections, 2011 IEEE International Symposium on Biomedical Imaging: From Nano to Macro, 2011, 512-515.

[5] X.S. Zhou, A. Gupta and D. Comaniciu, An information fusion framework for robust shape tracking, IEEE Transactions on Pattern Analysis and Machine Intelligence 27 (2005), 115-129.

[6] B. Georgescu, X.S. Zhou, D. Comaniciu and B. Rao, Real-time multi-model tracking of myocardium in echocardiography using robust information fusion, International Conference on Medical Image Computing and Computer Assisted Intervention 3216 (2004), 777-785.

[7] B.V. Ginneken, A.F. Frangi, J.J. Staal, B.M.H. Romeny and M.A. Viergever, Active shape model segmentation with optimal features, IEEE Transactions on Medical Imaging 21 (2002), 924-933.

[8] T. Heimann and H. Meinzer, Statistical shape models for 3D medical image segmentation: A review, Medical Image Analysis 13 (2009), 543-563.

[9] S.C. Mitchell, J.G. Bosch, B.P. Lelieveldt, R.J. Geest and J.H. Reiber, 3-D active appearance models: Segmentation of cardiac MR and ultrasound images, IEEE Transactions on Medical Imaging 21 (2002), 1167-1178.

[10] M. Dorigo and L. Gambardella, Ant colony system: A cooperative learning approach to the traveling salesman problem, IEEE Transactions on Evolutionary Computation 1 (1997), 53-66.

[11] Y.N. Zhang and L. Yang, Estimation of motion vectors in 3D ultrasound images using ant colony optimization, 4th International Conference on BioMedical Engineering and Informatics 2 (2011), 950-954.

[12] Y.-N. Z., Y. Le and K. Yan, Application of ant colony optimization in estimating motion vectors of ultrasound images, Journal of Northeastern University (Natural Science) 33 (2012), 327-331. 